\title{
Near infrared light variations of CP stars
}

\section{The SiSrCrEu stars ${ }^{\star}$}

\author{
F.A. Catalano ${ }^{1,3}$, F. Leone ${ }^{2,3}$, and R. Kroll ${ }^{4}$ \\ 1 Istituto di Astronomia, Città Universitaria, Viale A, Doria 6, I-95125 Catania, Italy \\ 2 Osservatorio Astrofisico di Catania, Città Universitaria, Viale A, Doria 6, I-95125 Catania, Italy \\ 3 C.N.R.-G.N.A, Unità di ricerca di Catania, Città Universitaria, Viale A, Doria 6, I-95125 Catania, Italy \\ ${ }^{4}$ Instituto de Astrofisica de Canarias, 38200 La Laguna, Tenerife, Spain
}

Received November 17, 1997; accepted February 4, 1998

\begin{abstract}
Twelve magnetic Chemically Peculiar (CP2) stars of the $\mathrm{SiSrCrEu}$ subgroup mostly brighter than the 7.5 visual magnitude have been investigated in the infrared at $1.25,1.6$ and $2.2 \mu$. The stars HD 74521, HD 90044, HD 119419, HD 125630, and HD 187473 are clearly variable in the near infrared with the same period as the visible light, spectrum, and magnetic field variations.

The stars HD 10783, HD 12447, HD 116458, HD 147010, HD 166469, HD 170397, and HD 223640 do show a smaller amount of variability, although with quite large a dispersion of the data.

A remarkable result of the present investigation is that, at least for the stars for which contemporaneous observations are available, the observed infrared variations appear to be in phase with the variations in the long wavelength part of the visible. This may be an indication that the mechanism of the infrared variations should be the same as for the visible.
\end{abstract}

Key words: stars: chemically peculiar, stars: variables infrared: stars

\section{Introduction}

Catalano et al. (1991, hereafter CKL) have shown that, out of the eight CP stars monitored throughout their rotational periods, at least six are variable in the near infrared, although with smaller amplitudes than in the visible.

On the basis of this result, we have started an observational campaign to search for infrared variability aimed

Send offprint requests to: F.A. Catalano

* Based on observations collected at the European Southern Observatory, La Silla, Chile.
Table 1. The list of the runs and the symbols used to represent in the figures the observations collected in different runs

\begin{tabular}{|clcc|}
\hline Run N. & Dates & symbol & observer \\
\hline 1 & 1986 Oct. $13-23$ & $\square$ & RK \\
2 & 1987 Nov. $1-8$ & $\nabla$ & FAC \\
3 & 1989 Apr. $19-26$ & $\circ$ & FAC \\
4 & 1991 Mar. $23-31$ & $\bullet$ & FAC \\
5 & 1991 Oct. $19-31$ & $\times$ & FAC \\
6 & 1993 Jan. $19-31$ & $\mathbf{\square}$ & FAC \\
7 & 1993 Oct. $1-11$ & $*$ & FAC \\
\hline
\end{tabular}

to test the reliability of the common idea that the infrared region is not affected by all those phenomena that characterise the ultraviolet and visible parts of CP star spectra and to better understand the origin of the light variability, which is one of the outstanding observational aspects of some subgroups of CP stars.

In a previous paper (Catalano et al. 1997), the first in a series devoted to the investigation of the infrared variability of CP stars, we have shown that, out of the twenty observed CP2 stars of the subgroup $\mathrm{SrEuCr}, 19$ have been found variable in the infrared and only one, i.e. HD 137949, was found to be non variable, at least on a time scale of a few years.

As it was stressed in Catalano et al. (1997), the variability of CP stars is currently explained in terms of the oblique rotator model, according to which, the period of the observed light, spectrum and magnetic field variations is nothing else than the rotational period, therefore accurate knowledge of the period of variability of CP stars is a fundamental step in understanding their complex behaviour, especially as far as it concerns the phase relation between the magnetic, spectral and light variations. Moreover the infrared variations of $\mathrm{CP}$ stars do show 
smaller amplitudes than in the visible, hence we mainly selected bright stars, not fainter than about $7.5 \mathrm{mag}$, and for which a period was previously known.

In this paper we report the results concerning twelve CP2 stars of the subgroup showing overabundances of $\mathrm{Si}$ and other elements.

\section{Observations}

The observations have been carried out in the near IR bands $J, H$, and $K$ at the $1 \mathrm{~m}$ photometric telescope at ESO, La Silla, Chile, using an InSb detector cooled with liquid nitrogen. The data have been collected during several observing runs from July 1986 through January 1993. The list of the observing runs is given in Table 1 together with the symbols used to identify the observations made in each run. Indeed during the run of March 1991 contemporaneous uvby photometry has been carried out at the Danish $50 \mathrm{~cm}$ telescope, whose results have been published elsewhere (Catalano \& Leone 1993). All program stars were measured relative to closeby comparisons, which were chosen to have as similar colour and brightness as possible. The program stars and their comparisons are listed in Table 2. Central wavelengths and bandwidths (in $\mu \mathrm{m}$ ) of the used filters are: $J: 1.24,0.32$; $H: 1.63,0.28 ; K: 2.19,0.39$.

In the 1986 campaign the integration times were selected as to give a photon noise precision of 0.01 mag or better, during the subsequent runs the integration times, the number of cycles, and the desired rms accuracy in the mean level were optimised to get a $2 \%$ maximum error in the observations: the resulting accuracy in the final reduced data is typically $0.006 \mathrm{mag}$.

A detailed description of the ESO infrared photometers can be found in Bouchet (1989). ESO standard software was used for all reduction steps. Magnitudes in the standard IR system have also been obtained by observing suitable standard stars from the ESO list (Bouchet et al. 1991).

\section{Light curves of individual stars}

The adopted ephemeris elements of the infrared light curves for the program stars are listed in Table 3; they have been mainly taken from Catalano \& Renson (1984, 1988, 1997); Catalano et al. (1991, 1993), and references therein. For each light curve a least square fit of all the data has been performed with a function of the type:

$$
\begin{aligned}
\Delta m=A_{0} & +A_{1} \sin 2 \pi\left[\left(t-t_{0}\right) / P+\phi_{1}\right] \\
& +A_{2} \sin 2 \pi\left[2\left(t-t_{0}\right) / P+\phi_{2}\right] .
\end{aligned}
$$

In this relation $\Delta m$ is the magnitude difference in each filter between the $\mathrm{CP}$ star and the comparison star, $t$ is the JD date, $t_{0}$ is the adopted initial epoch, $P$ is the period in days. This procedure can be partially accounted for by considering that within the accuracy of the measurements a sine wave and its first harmonic appear to be generally adequate to describe the light curves (North 1984; Mathys \& Manfroid 1985) and the magnetic field variations (Borra \& Landstreet 1980; Bohlender et al. 1993).

In the figures, where the infrared variations are plotted versus the ephemeris elements adopted for each program star (and summarised in Table 3), open squares represent the data collected in the 1986 run (i.e. the data with an accuracy lower than $0.01 \mathrm{mag}$ ), while the other symbols, listed in Table 1, represent the observations of the successive runs having an accuracy better than 0.01 mag. In the same figures the continuous line represents the fit to the observations obtained by means of Eq. (1): indeed this fit, whose coefficients and the sigma are presented in the last three columns of Table 3, has to be considered only as indicative, just to evidentiate the observed variations. It has to be noted here that, in spite of the fact that for some stars the infrared observations span long time intervals, the observed variations show lower amplitudes than in the visible and larger dispersions too, so that the selection between the aliases in the period values is not reliable and very often it does not remove at all the uncertainties. For these reasons we preferred to rest on the visible light curves, which generally show larger amplitudes and lower dispersion. However for most of the stars the individual fits of the $J, H$, and $K$ variations did show very similar behaviour, and in many cases the first harmonic was sufficient to fit adequately the observations.

\section{$H D 10783=G C 2141=U Z P s c$}

The light variability of HD 10783 was detected by Van Genderen (1964) who preferred the value $4.1565 \mathrm{~d}$ of the period to the value $4.134 \mathrm{~d}$ derived by Steinitz (1964) from the analysis of Babcock's (1958) effective magnetic field data. $U B V$ photometry and magnetic field measurements were carried out by Preston \& Stepien (1968), who refined the period to the value $4.1327 \mathrm{~d}$. Additional photometric observations by Van Genderen $(1967,1971)$ have shown the photometric period to be consistent with the value determined by Preston \& Stepien (1968).

Recent photometric $U B V$ observations have been carried out by Hardie et al. (1990), who have derived the ephemeris elements given in Table 3, which have been adopted to plot our infrared observations in Fig. 1. From this figure a small variation (with an amplitude of the order of $0.02 \mathrm{mag}$ ) is evident in all three filters, although with quite a large dispersion.

$H D 12447=H R 596=\alpha^{2} P s c$

HD 12447 is the brightest component of a visual binary system (ADS 1615) whose secondary (0.9 mag fainter) is only $3.6^{\prime \prime}$ apart. 
Table 2. Program stars, comparison stars, and their characteristics. Spectral types for the program stars are taken from the General Catalogue of CP stars (Renson et al. 1991), those of comparison stars are from the Bright Star Catalogue (Hoffleit \& Jaschek 1982). IR magnitudes are mean values from the present observations

\begin{tabular}{|c|c|c|c|c|c|c|c|c|c|c|c|c|c|}
\hline \multicolumn{7}{|c|}{ Program Stars } & \multicolumn{7}{|c|}{ Comparison Stars } \\
\hline $\mathrm{HD}$ & $\mathrm{HR} / \mathrm{GC}$ & Sp. type & $m_{V}$ & $\langle J\rangle$ & $<H>$ & $<K>$ & $\mathrm{HD}$ & $\mathrm{HR} / \mathrm{GC}$ & Sp. type & $m_{V}$ & $\langle J\rangle$ & $<H>$ & $<K>$ \\
\hline 10783 & GC 2141 & $\mathrm{~A} 2 \mathrm{SiCrSr}$ & 6.55 & 6.609 & 6.630 & 6.626 & 10761 & HR 510 & G8III & 4.36 & 2.731 & 2.251 & 2.145 \\
\hline 12447 & HR 596 & A2 SiSrCr & 4.22 & 3.768 & 3.755 & 3.739 & 12573 & HR 607 & $\mathrm{~A} 3 \mathrm{~m}$ & 5.56 & 5.141 & 5.068 & 5.034 \\
\hline 74521 & HR 3465 & $\mathrm{~A} 1 \mathrm{SiEuCr}$ & 5.65 & 5.802 & 5.861 & 5.878 & 74783 & HR 3481 & $\mathrm{~A} 1 \mathrm{~V}$ & 5.87 & 5.693 & 5.646 & 5.613 \\
\hline 90044 & HR 4082 & B9 SiCrSr & 5.96 & 6.057 & 6.111 & 6.113 & 90882 & HR 4116 & B9.5 V & 5.21 & 5.266 & 5.301 & 5.296 \\
\hline 116458 & HR 5049 & $\mathrm{~A} 0 \mathrm{SiEuCr}$ & 5.67 & 5.702 & 5.725 & 5.729 & 115967 & HR 5030 & $\mathrm{~B} 6 \mathrm{~V}$ & 6.05 & 5.873 & 5.851 & 5.834 \\
\hline 119419 & HR 5158 & A0 $\mathrm{SiCrEu}$ & 6.46 & 6.659 & 6.732 & 6.748 & 117150 & HR 5071 & $\mathrm{~A} 1 \mathrm{~V}$ & 5.06 & 4.856 & 4.825 & 4.796 \\
\hline 125630 & GC 19369 & A $2 \mathrm{SiCrSr}$ & 6.79 & 6.738 & 6.743 & 6.729 & 125990 & HR 5382 & A3 V & 6.36 & 6.104 & 6.039 & 6.003 \\
\hline 147010 & GC 21960 & B9 $\mathrm{SiCrSr}$ & 7.39 & 6.844 & 6.789 & 6.749 & 146284 & GC 21873 & B8 IV & 6.67 & 6.215 & 6.146 & 6.099 \\
\hline 166469 & HR 6802 & A0 $\mathrm{SiCrSr}$ & 6.51 & 6.474 & 6.483 & 6.468 & 167666 & HR 6835 & A7 V: & 6.19 & 5.771 & 5.695 & 5.653 \\
\hline 170397 & HR 6932 & A0 SiCrEu & 6.03 & 6.006 & 6.016 & 6.005 & 171130 & HR 6962 & $\mathrm{~A} 2 \mathrm{~V}$ & 5.76 & 5.716 & 5.712 & 5.696 \\
\hline 187473 & GC 27467 & B9 EuCrSi & 7.32 & 7.426 & 7.459 & 7.464 & 190285 & GC 27842 & A0 V & 7.24 & 7.060 & 7.028 & 6.998 \\
\hline 223640 & HR 9031 & B9 $\mathrm{SiSrCr}$ & 5.18 & 5.4328 & 5.513 & 5.540 & 222847 & HR 8998 & $\mathrm{~B} 8 \mathrm{~V}$ & 5.24 & 5.391 & 5.422 & 5.423 \\
\hline
\end{tabular}

Table 3. Ephemeris elements adopted to compute the phases of the variations, coefficients of the fits and error

\begin{tabular}{|c|c|c|c|c|c|c|c|}
\hline HD & $\mathrm{JD}(\phi=0)$ & Instant of & $P($ days $)$ & coeff. & $J$ & $H$ & $K$ \\
\hline 10783 & 2439758.002 & $B \max$. & 4.13281 & $\begin{array}{c}A_{1} \\
A_{2} \\
\sigma\end{array}$ & $\begin{array}{c}0.0100 \\
- \\
0.0099\end{array}$ & $\begin{array}{c}0.0057 \\
- \\
0.0087\end{array}$ & $\begin{array}{c}-0.0079 \\
- \\
0.0089\end{array}$ \\
\hline 12447 & 2443117.822 & first magn. obs. & 1.49070 & $\begin{array}{c}A_{1} \\
A_{2} \\
\sigma\end{array}$ & $\begin{array}{l}0.0015 \\
0.0025 \\
0.0106\end{array}$ & $\begin{array}{l}0.0039 \\
0.0030 \\
0.0063\end{array}$ & $\begin{array}{l}0.0045 \\
0.0033 \\
0.0074\end{array}$ \\
\hline 74521 & 2448344.539 & uvby max. & 7.76851 & $\begin{array}{c}A_{1} \\
A_{2} \\
\sigma\end{array}$ & $\begin{array}{l}0.0086 \\
0.0085 \\
0.0060\end{array}$ & $\begin{array}{l}0.0069 \\
0.0077 \\
0.0023\end{array}$ & $\begin{array}{l}0.0063 \\
0.0042 \\
0.0055\end{array}$ \\
\hline 90044 & 2445659.000 & $u$ min. & 4.37900 & $\begin{array}{c}A_{1} \\
A_{2} \\
\sigma\end{array}$ & $\begin{array}{c}0.0153 \\
- \\
0.0054\end{array}$ & $\begin{array}{c}0.0162 \\
- \\
0.0076\end{array}$ & $\begin{array}{c}0.0161 \\
- \\
0.0057\end{array}$ \\
\hline 116458 & 2448341.719 & $u \max$. & 4.27349 & $\begin{array}{c}A_{1} \\
A_{2} \\
\sigma\end{array}$ & $\begin{array}{c}0.0068 \\
- \\
0.0094\end{array}$ & $\begin{array}{c}0.0046 \\
- \\
0.0060\end{array}$ & $\begin{array}{c}0.0073 \\
- \\
0.0053\end{array}$ \\
\hline 119419 & 2448340.711 & uvby max. & 2.60562 & $\begin{array}{c}A_{1} \\
A_{2} \\
\sigma\end{array}$ & $\begin{array}{c}0.0090 \\
- \\
0.0065\end{array}$ & $\begin{array}{c}0.0086 \\
- \\
0.0049\end{array}$ & $\begin{array}{c}0.0086 \\
- \\
0.0072\end{array}$ \\
\hline 125630 & 2448344.752 & $y \max$. & 2.20552 & $\begin{array}{c}A_{1} \\
A_{2} \\
\sigma\end{array}$ & $\begin{array}{c}0.0397 \\
\overline{0} \\
0.0249\end{array}$ & $\begin{array}{c}0.0333 \\
-\overline{0184}\end{array}$ & $\begin{array}{c}0.0212 \\
-\overline{0175}\end{array}$ \\
\hline 147010 & 2448346.859 & $u v b y \min$. & 3.9206763 & $\begin{array}{l}A_{1} \\
A_{2}\end{array}$ & 0.0074 & 0.0111 & 0.0068 \\
\hline 166469 & 2448342.891 & uvby max. & 2.88632 & $\begin{array}{c}\sigma \\
A_{1} \\
A_{2} \\
\sigma\end{array}$ & $\begin{array}{c}0.0096 \\
0.0080 \\
- \\
0.0114\end{array}$ & $\begin{array}{c}0.0087 \\
0.0086 \\
- \\
0.0094\end{array}$ & $\begin{array}{c}0.0086 \\
0.0075 \\
-\overline{0140}\end{array}$ \\
\hline 170397 & 2448341.827 & $u$ min. & 2.19133 & $\begin{array}{c}\sigma \\
A_{1} \\
A_{2} \\
\sigma\end{array}$ & $\begin{array}{c}0.0067 \\
-\overline{0} \\
0.0076\end{array}$ & $\begin{array}{c}0.0060 \\
-\overline{0} \\
0.0047\end{array}$ & $\begin{array}{c}0.0052 \\
- \\
0.0076\end{array}$ \\
\hline 187473 & 2442619.578 & $\phi=0$ & 4.718 & $\begin{array}{c}A_{1} \\
A_{2} \\
\sigma\end{array}$ & $\begin{array}{l}0.0350 \\
0.0273 \\
0.0070\end{array}$ & $\begin{array}{l}0.0341 \\
0.0305 \\
0.0063\end{array}$ & $\begin{array}{l}0.0335 \\
0.0308 \\
0.0007\end{array}$ \\
\hline 223640 & 2444696.820 & $B_{\text {eff }} \max$. & 3.735239 & $\begin{array}{c}A_{1} \\
A_{2} \\
\sigma\end{array}$ & $\begin{array}{c}0.0036 \\
- \\
0.0114\end{array}$ & $\begin{array}{c}0.0100 \\
- \\
0.0099\end{array}$ & $\begin{array}{c}0.0082 \\
- \\
0.0111\end{array}$ \\
\hline
\end{tabular}

HD 12447 has been found variable in light by Winzer (1974) with a period of $0.7383 \mathrm{~d}$, which later was inferred by Borra \& Landstreet (1980) to be probably due to the variability of the comparison star HD 13467, in fact Borra \& Landstreet found their longitudinal magnetic field measurements to be compatible with period values such as: $0.5920,0.5975,0.6042,0.7508,1.45050,1.49070$, $1.5257,2.7552,2.8764$, and $3.0395 \mathrm{~d}$, and preferred the value $1.49070 \mathrm{~d}$ which gave the smallest $\chi^{2} / \nu$ ratio.
In spite of the fact that the infrared observations of HD 12447 span a longer time interval than the magnetic data, the small amplitudes and the quite large dispersion of the observed variations prevented any selection between the aliases in the period values. On the other hand the magnetic variation as observed by Borra \& Landstreet is well defined, so that we adopted their period value.

Our infrared observations of HD 12447 are plotted in Fig. 2 versus the phase computed by means of Borra \& Landstreet (1980) ephemeris elements listed in Table 3: 


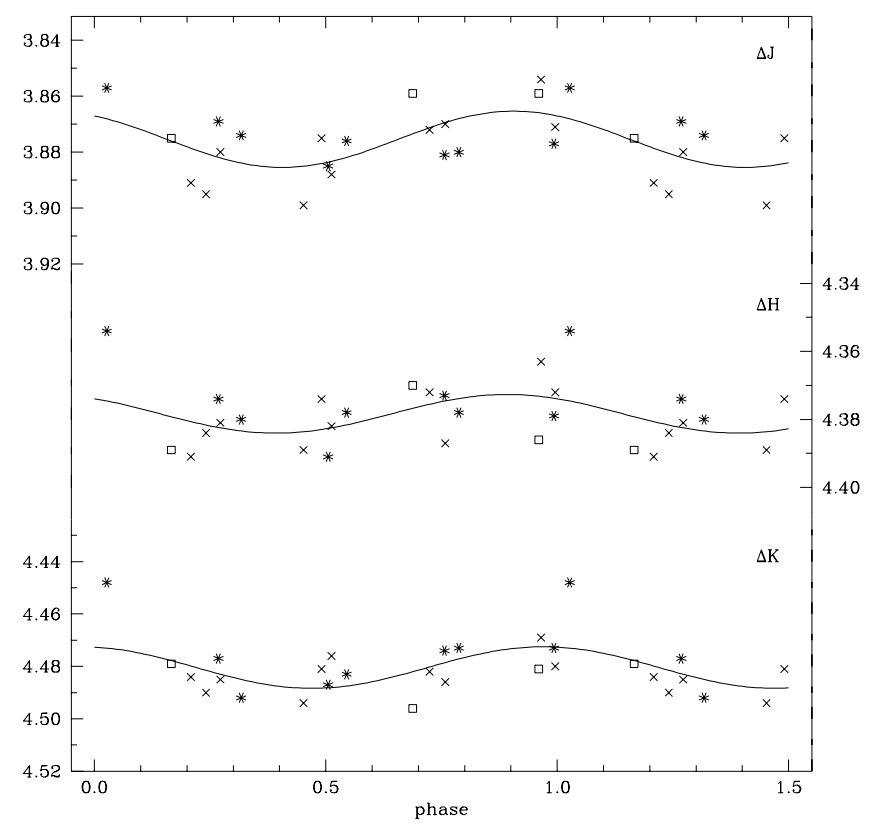

Fig. 1. Infrared light curves of HD 10783. The phases are computed according to the ephemeris elements given in Table 3. The solid line is a least-square fit of the observations by Eq. (1) as described in the text

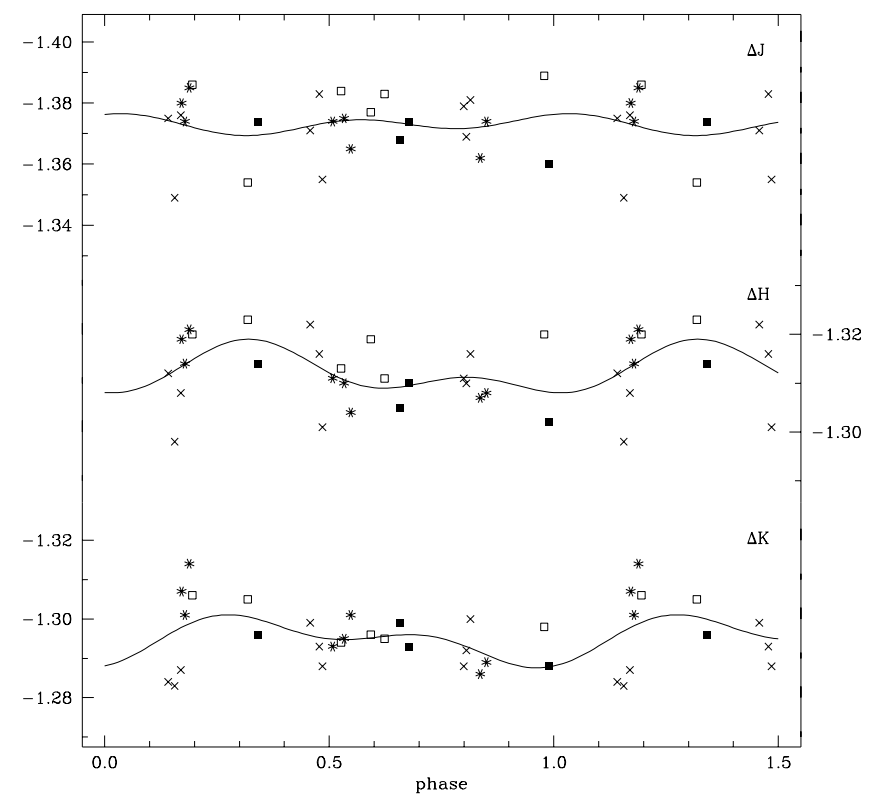

Fig. 2. Infrared light curves of HD 12447. The phases are computed according to the ephemeris elements given in Table 3. The solid line is a least-square fit of the observations by Eq. (1) as described in the text

the dispersion of the data is quite large and the period could also be somewhat uncertain. However the individual fits of the infrared variations appear better defined by a double harmonic in $H$ and $K$.
$H D 74521=H R 3465=49 C n c=B I C n c$

HD 74521 has been discovered to be a spectrum variable by Deutsch (1947). The first photometric observations were carried out in $U B V$ by Stepien (1968) who derived a period of $5.43 \mathrm{~d}$.

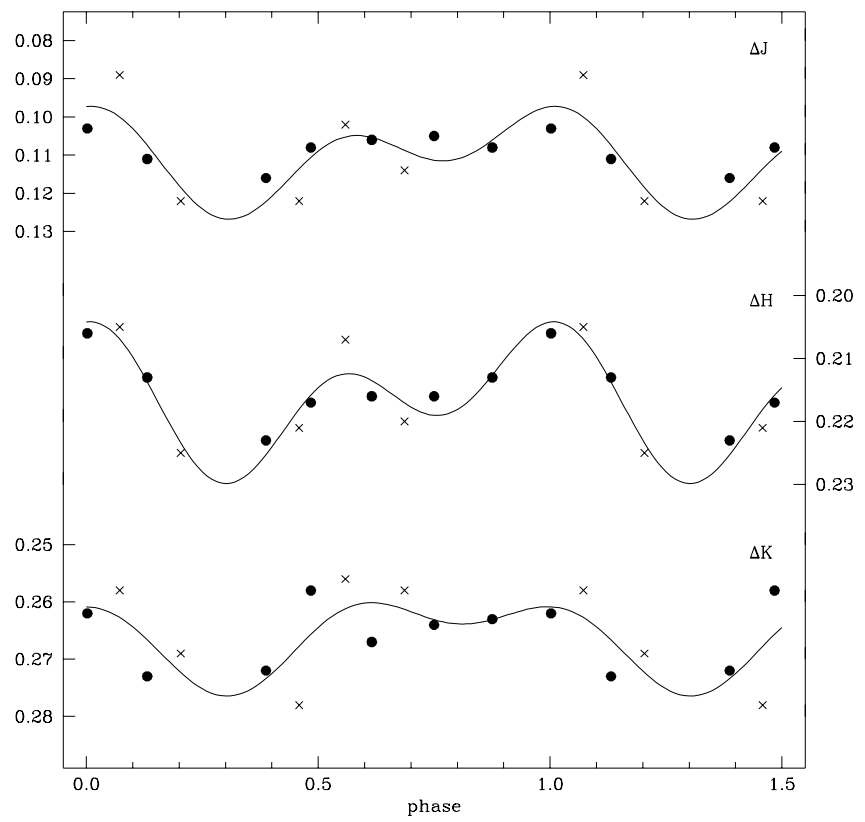

Fig. 3. Infrared light curves of HD 74521. The phases are computed according to the ephemeris elements given in Table 3. The solid line is a least-square fit of the observations by Eq. (1) as described in the text

From subsequent $U B V$ observations Winzer (1974) showed this period to be incorrect, and found the best representation of his data was obtained with a period of 4.2359 d. Later on Rakos \& Fiedler (1978) found a nearby value of $4.239 \mathrm{~d}$. From photometric observations in uvby and spectrophotometry Adelman \& Pyper (1979) found the colour indices of HD 74521 to vary but they could not refine the period. On the basis of photometric observations in the Geneva system Lanz \& Mathys (1991) found the most probable period to be $7.769( \pm 0.001) \mathrm{d}$.

The longitudinal magnetic field of HD 74521 was first measured by Babcock (1958), who found it variable in the range from -180 to +1450 gauss. Mathys (1991) has carried out further magnetic observations and by comparing his own data together with those by Babcock (1958) and by Bohlender \& Landstreet (1991), found the best representation of the longitudinal magnetic field data to occur with periods of the order of 7.7730 or $1.1475 \mathrm{~d}$ (which is close to the $1 \mathrm{~d}^{-1}$ alias of the former value). According to Mathys' analysis the longitudinal magnetic field varies in the range 500 to 800 gauss and does not show reversal of polarity.

From recent uvby observations Catalano \& Leone (1993) got the best representation of all sets of data by 
means of the period value: $7.76851( \pm 0.00015) \mathrm{d}$, which also gives quite a good representation of the magnetic field data.

The infrared light curves of HD 74521 are plotted in Fig. 3 versus the phase computed by means of of Catalano \& Leone (1993) ephemeris elements listed in Table 3. From this figure we see that the infrared variations are doublewaved and all in phase with each other. The amplitude is almost the same $(\sim 0.02 \mathrm{mag})$ in all filters. The behaviour of the light curves is also the same in all filters.

$H D 90044=H R 4082=25 S e x=S S S e x$

The spectrum variability of HD 90044 has been discovered by Bonsack (1974), who found variations of the SrII and CaII lines with full observed range occurring on spectrograms taken two days apart.

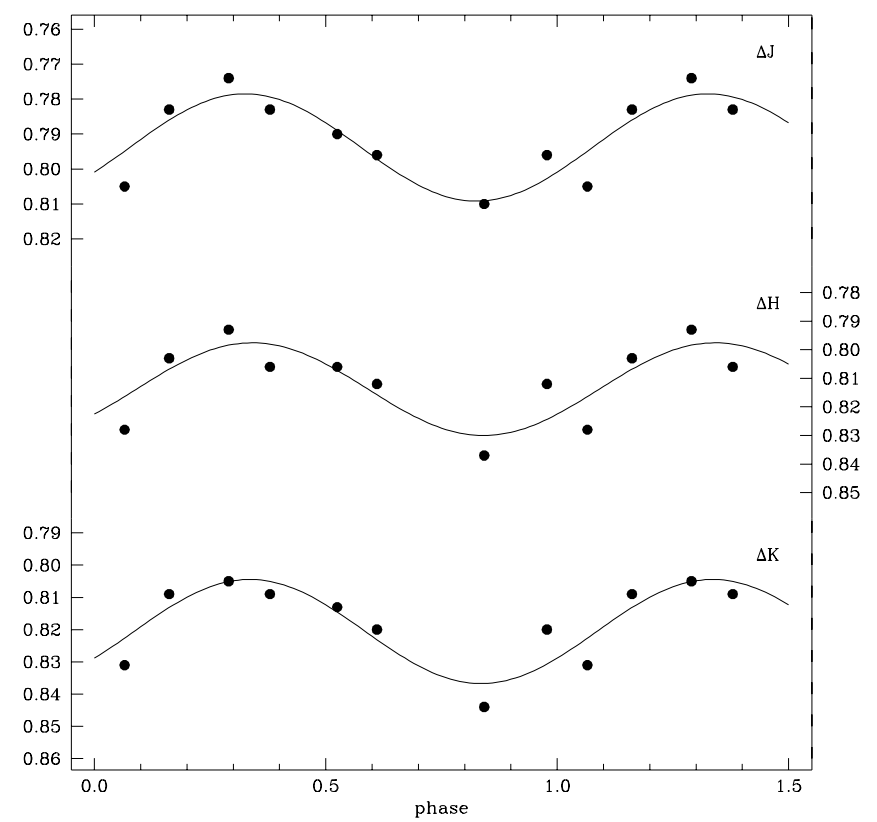

Fig. 4. Infrared light curves of HD 90044. The phases are computed according to the ephemeris elements given in Table 3 . The solid line is a least-square fit of the observations by Eq. (1) as described in the text

The light variability of HD 90044 has been studied by Manfroid \& Renson $(1980,1983)$ who found the period to be $4.37 \mathrm{~d}$, value confirmed later by Mathys \& Manfroid (1985).

Longitudinal magnetic field observations have been carried out by Bohlender et al. (1993) who considered the detection of a field fairly certain although they could not construct a phase diagramme because of the large uncertainty in the period.

Recent refinements of the period of HD 90044 have been performed by Catalano \& Leone (1993) and Manfroid
\& Renson (1994) by using their own uvby observations together to the data collected in the Long-Term Photometry of Variables Project (Manfroid et al. 1991).

The infrared light curves of HD 90044 are plotted in Fig. 4 versus the phase computed on the basis of the ephemeris elements taken from Manfroid \& Renson (1994) and listed in Table 3. From Fig. 4 we see that the infrared variations of HD 90044 are single-waved and all in phase with each other. The amplitude is almost the same $(\sim 0.03 \mathrm{mag})$ in all filters.

\section{HD $116458=$ HR $5049=67 G$. Mus}

HD 116458 is a very interesting sharp-lined CP2 star: in fact it has one of the highest known absolute values of the $\lambda 5200$ photometric index $\Delta a(0.054)$.

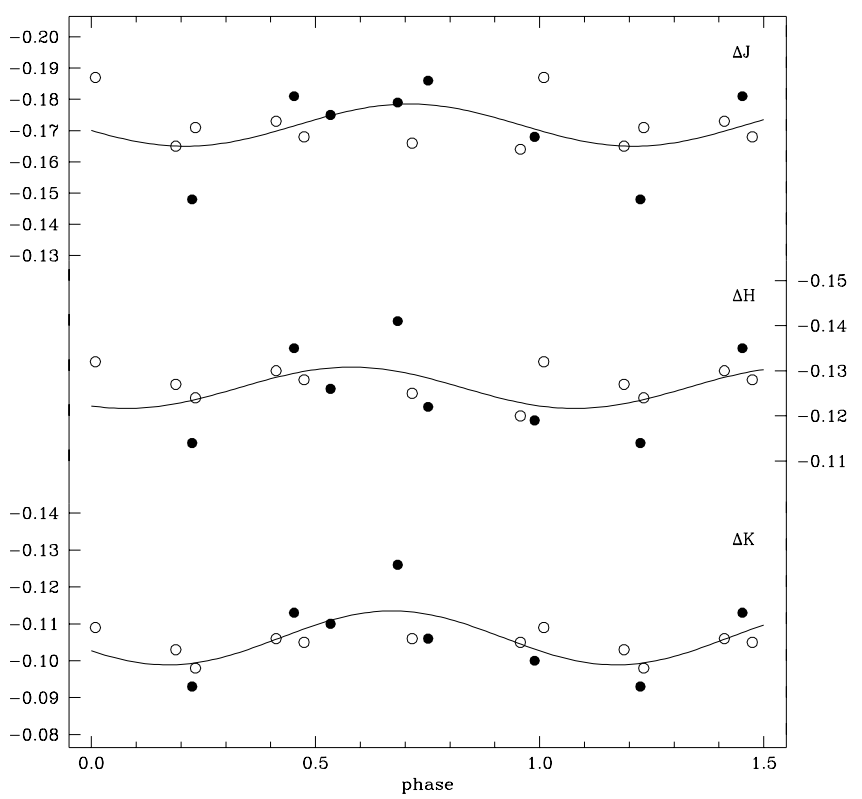

Fig. 5. Infrared light curves of HD 116458. The phases are computed according to the ephemeris elements given in Table 3. The solid line is a least-square fit of the observations by Eq. (1) as described in the text

From the fact that very different spectral classification are available in the literature, Dworetsky et al. (1980) found entirely possible that this star is a fairly extreme spectrum variable.

Wood \& Campusano (1975) measured a strong negative longitudinal magnetic field and supported evidence it be a spectroscopic binary with a period of $70.651 \mathrm{~d}$. Albrecht et al. (1977) found the magnetic field do not differ much from a mean value of about -1800 gauss except for two deviating values (out of twelve), and confirmed the spectroscopic binarity with the above mentioned value of the period. A preliminary orbit with a period of $70.651 \mathrm{~d}$ and a large eccentricity $(e \approx 0.4)$ has been determined by 
Maitzen \& Wood (1977) who also found HD 116458 to be constant in light and colour over a time scale of the order of $10 \mathrm{~d}$, in accordance with the fact that longer periods tend to occur for larger $\lambda 5200$ depression stars.

Mathys (1991) measured the longitudinal magnetic field of HD 116458 and supported evidence for this star having an essentially constant negative magnetic field of the order of -2000 gauss, though low amplitude variations (of the order of ten percent) could be present in a long time scale.

More recently Hensberge (1993) inferred a period value of $147.9 \pm 0.6$ from light and spectrum variations. However by combining their uvby observations with those collected in the Long-Term Photometry of Variables Project (Manfroid et al. 1991); Catalano \& Leone (1993) found all photometric data to be well represented with such a short period as $4.27349( \pm 0.00032) \mathrm{d}$.

The infrared light curves of HD 116458 are plotted in Fig. 5 versus the phase computed by means of Catalano \& Leone (1993) ephemeris elements listed in Table 3. The infrared variations of HD 116458 have very small amplitudes, of the order of $0.01 \mathrm{mag}$ and are all in phase with each other, but the period has to be confirmed.

\section{HD $119419=$ HR $5158=$ V827 Cen}

The light variations of HD 119419 have been first observed in the uvby photometric system by Manfroid \& Renson $(1980,1983)$ who found a period of 2.605 d. Later Manfroid \& Mathys (1985) confirmed this value of the period. By means of new measurements in the Geneva system Lanz \& Mathys (1991) improved the period to the value: $2.6006( \pm 0.0003) \mathrm{d}$.

More recently Catalano \& Leone (1993) got a period value of $2.60562( \pm 0.00008) \mathrm{d}$.

A strong polarity reversing longitudinal magnetic field has been discovered by Thompson et al. (1987). Indeed Mathys (1991) finds HD 119419 to be one of the few stars showing definite evidence of an anharmonic variation of the magnetic field. From line intensity measurements Mathys also finds the equivalent width of FeII $\lambda 5961$ and SiII $\lambda 5978$ lines to vary in phase with each other and nearly in anti-phase with respect to the magnetic field.

The infrared light curves of HD 119419 are plotted in Fig. 6 versus the phase computed by means of Catalano \& Leone (1993) ephemeris elements listed in Table 3. The analysis of the infrared light curves shows that all of them are single-waved and in phase with each other, with amplitudes of the order of $0.02 \mathrm{mag}$.

$$
H D 125630=G C 19369=B S C i r
$$

The star HD 125630 has been discovered to be variable in light with a period $P=2.205 \mathrm{~d}$ by Manfroid \& Renson (1980, 1983).

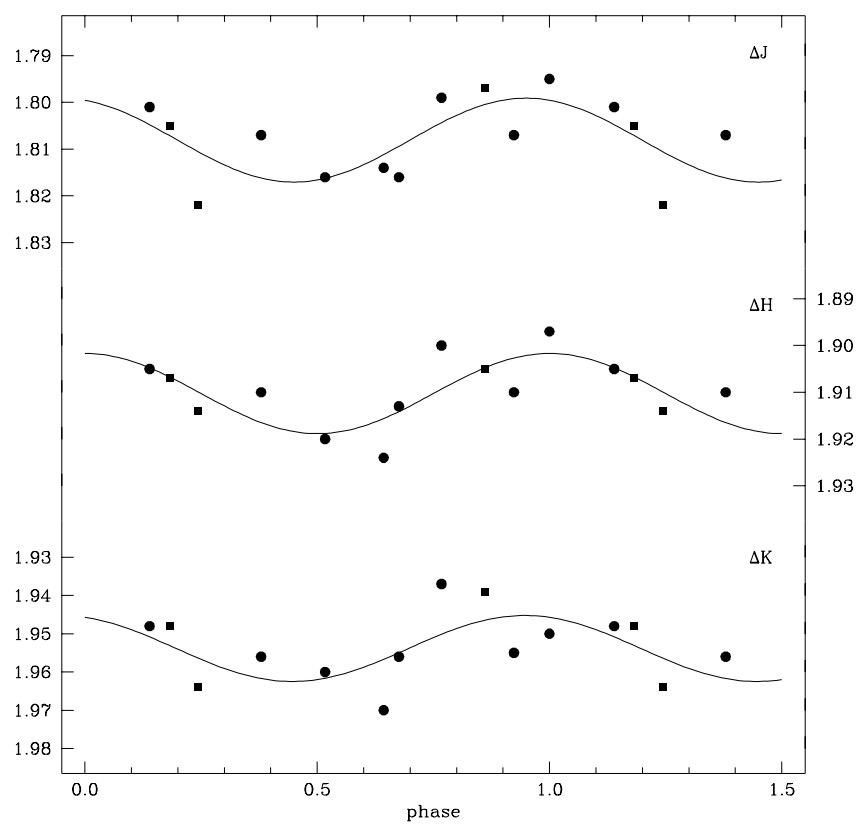

Fig. 6. Infrared light curves of HD 119419. The phases are computed according to the ephemeris elements given in Table 3. The solid line is a least-square fit of the observations by Eq. (1) as described in the text

This value of the period was later on confirmed by Mathys \& Manfroid (1985) who reanalysed the same set of data. Recent uvby observations have been carried out by Catalano \& Leone (1993) leading to the improved period value: $2.20552( \pm 0.00006) \mathrm{d}$.

No spectroscopic study nor magnetic field determination are available for this star.

The infrared light curves of HD 125630 are plotted in Fig. 7 versus the phase computed by means of Catalano \& Leone (1993) ephemeris elements listed in Table 3. As it is evident from Fig. 7, the infrared light curves of HD 125630 show a simple-waved behaviour, with quite large but different amplitudes amounting to 0.08 mag in $J$ and $H$, and to 0.06 mag in $K$.

$H D 147010=G C 21960=B D-19^{\circ} 4359=$ V933 Sco

The star HD 147010 has been discovered to be spectrum variable by Kameswara Rao \& Rajamohan (1982) who found drastic changes in the strength of the CrII $\lambda 4012.5$ line and estimated the period to be of the order of $5.7 \mathrm{~d}$.

The light variability of this star has been ascertained by North $(1982,1984)$ who determined the period to be $3.9210( \pm 0.0001)$ d from photometric observations in the Geneva system.

From observations in the $v, b$, and $y$ filters of the Strömgren system Borra et al. (1985) found the period to be $3.99827 \mathrm{~d}$. Recently, on the basis of several new measurements in the Geneva system, Lanz \& Mathys (1991) have improved the period to the value 3.92076 


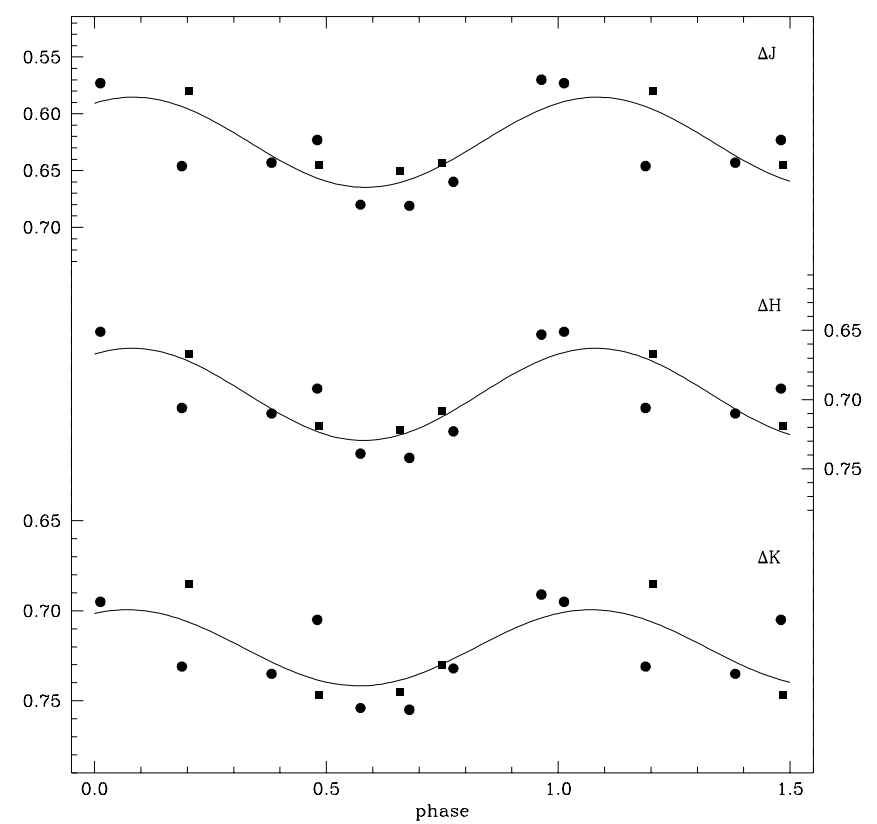

Fig. 7. Infrared light curves of HD 125630. The phases are computed according to the ephemeris elements given in Table 3. The solid line is a least-square fit of the observations by Eq. (1) as described in the text

$( \pm 0.00010)$ d. On the basis of recent uvby observations Catalano \& Leone (1993) have refined the period to the value: $3.920676( \pm 0.000005) \mathrm{d}$.

A very strong longitudinal magnetic field of negative polarity was measured independently by Brown et al. (1981) and Glagolevskii et al. (1982). Subsequently Thompson et al. (1987) studied the magnetic field variation of HD 147010 and found the period to be consistent with the photometric one derived by North (1982) and Borra et al. (1985). Mathys (1991) has confirmed the quite sinusoidal character without polarity reversal of the longitudinal magnetic field variation, occurring with the same period as the photometric one, and extrema in the range -2000 to -5000 gauss, in partial agreement with the measurements of Thompson et al. (1987) which appear to be more negative by about 1200 gauss. Mathys also studied the equivalent width of the FeII $\lambda 5961$ line and found large variations whose extrema coincide in phase with the light variations but do show no simple phase relation with the extrema of the magnetic field.

The infrared light curves of HD 147010 are shown in Fig. 8, where they are plotted versus the phase computed by means of Catalano \& Leone (1993) ephemeris elements listed in Table 3. From Fig. 8 we see that all light variations are single-waved and in phase with each other. The amplitude is almost the same in all filters amounting to about $0.02 \mathrm{mag}$.

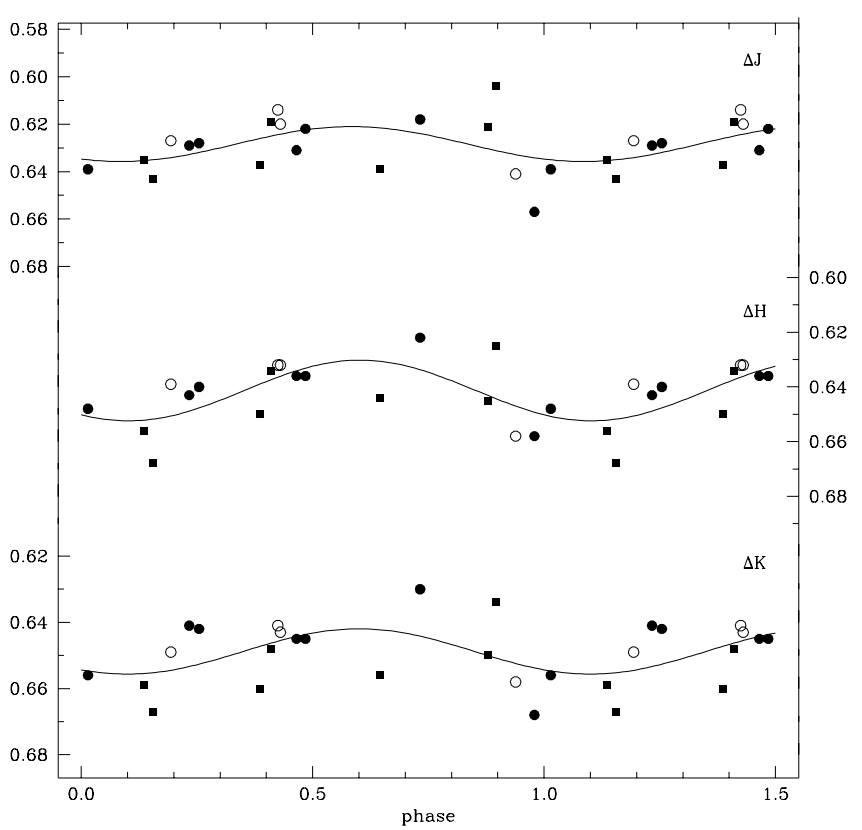

Fig. 8. Infrared light curves of HD 147010. The phases are computed according to the ephemeris elements given in Table 3. The solid line is a least-square fit of the observations by Eq. (1) as described in the text

$H D 166469=H R 6802=V 4045 \mathrm{Sgr}$

The star HD 166469 has been discovered to be variable in light by Renson (1978), who derived a period of 2.90 $( \pm 0.04) \mathrm{d}$.

From careful reexamination of the same set of data Manfroid \& Mathys (1985) derived a value of 2.8855 $( \pm 0.0008) \mathrm{d}$ or other nearby values. From recent uvby observations carried out at ESO, Catalano \& Leone (1993) have refined the period to the value: $2.88632( \pm 0.00015) \mathrm{d}$.

Nor spectroscopic neither magnetic studies are known for this star.

The infrared light curves of HD 166469 are plotted in Fig. 9 versus the phase computed by means of Catalano \& Leone (1993) ephemeris elements listed in Table 3. From Fig. 9 we see that the infrared light variations are singlewaved and in phase with each other with an almost constant amplitude of about $0.02 \mathrm{mag}$ or slightly smaller.

\section{$H D 170397=H R 6932=$ V432 Sct}

The star HD 170397 has been discovered to be variable in light by Renson (1978), who derived a period of 2.21 $( \pm 0.03) \mathrm{d}$.

From the same set of data Manfroid \& Mathys (1985) derived a value of $2.1912( \pm 0.0005)$ d or other nearby values. Comparing their recent uvby observations with those of Manfroid \& Mathys (1985); Catalano \& Leone (1993) have refined the period to the value: $2.19133( \pm 0.00005) \mathrm{d}$.

The longitudinal magnetic field of HD 170397 has been measured and found to be variable by Borra \& Landstreet 


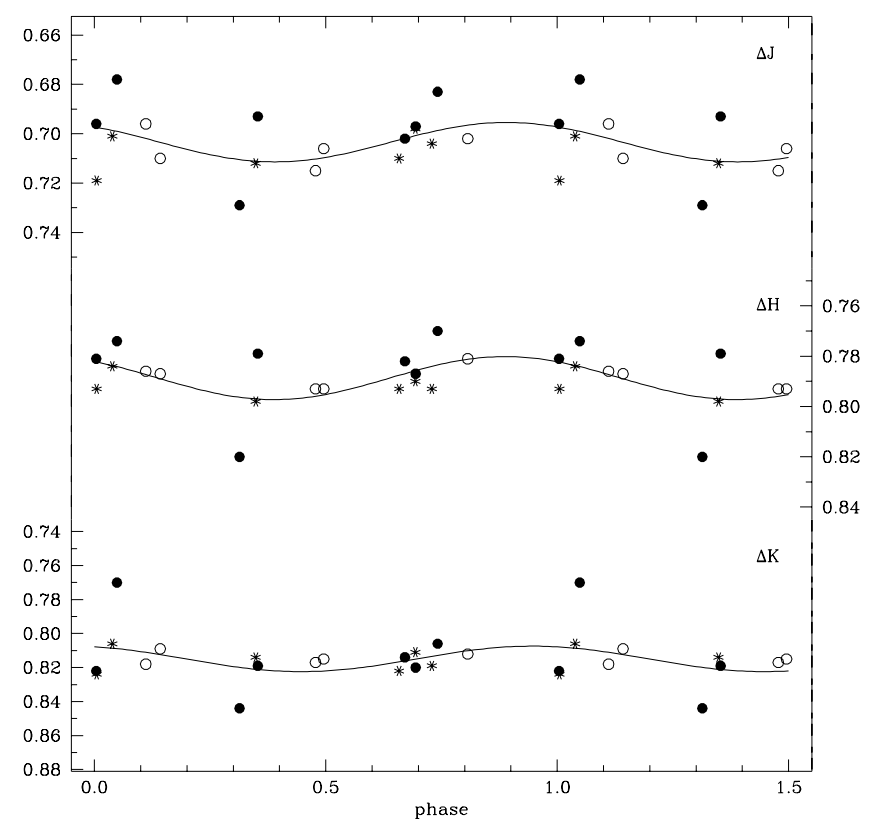

Fig. 9. Infrared light curves of HD 166469. The phases are computed according to the ephemeris elements given in Table 3. The solid line is a least-square fit of the observations by Eq. (1) as described in the text

(1980) who estimated the period to be $2.24 \mathrm{~d}$. Some more magnetic field measurements by Bohlender \& Landstreet (1991) and Mathys (1991) are well represented by the $2.1912 \mathrm{~d}$ period.

The infrared light curves of HD 170397 are plotted in Fig. 10 versus the phase computed by means of Catalano \& Leone (1993) ephemeris elements given in Table 3. From Fig. 10 we see that all light variations are single-waved and in phase with each other and the amplitude is of about $0.01 \mathrm{mag}$ in all filters.

$$
H D 187473=G C 27467=V 4064 S g r
$$

The variability of HD 187473 has been detected and studied by Hensberge et al. $(1977,1978)$, we found this star to vary with a period of $4.718 \pm 0.001 \mathrm{~d}$ showing extraordinary light variability with such large amplitudes as $\Delta u \leq 0.2 \mathrm{mag}, \Delta v=0.07 \mathrm{mag}$, and $\Delta b=\Delta y=0.1 \mathrm{mag}$ However, in spite of such very large range of light variations, no spectrum nor magnetic field observations have been carried out yet.

The infrared light curves of HD 187473 are plotted in Fig. 11 versus the phase computed by means of Hensberge et al. (1978) ephemeris elements listed in Table 3. From Fig. 11 we see that the infrared light curves are in phase with each other and show the same double-wave behaviour. In spite of the few observations, it is to be noted the outstanding amplitude, of the order of $0.1 \mathrm{mag}$, the largest observed until now.

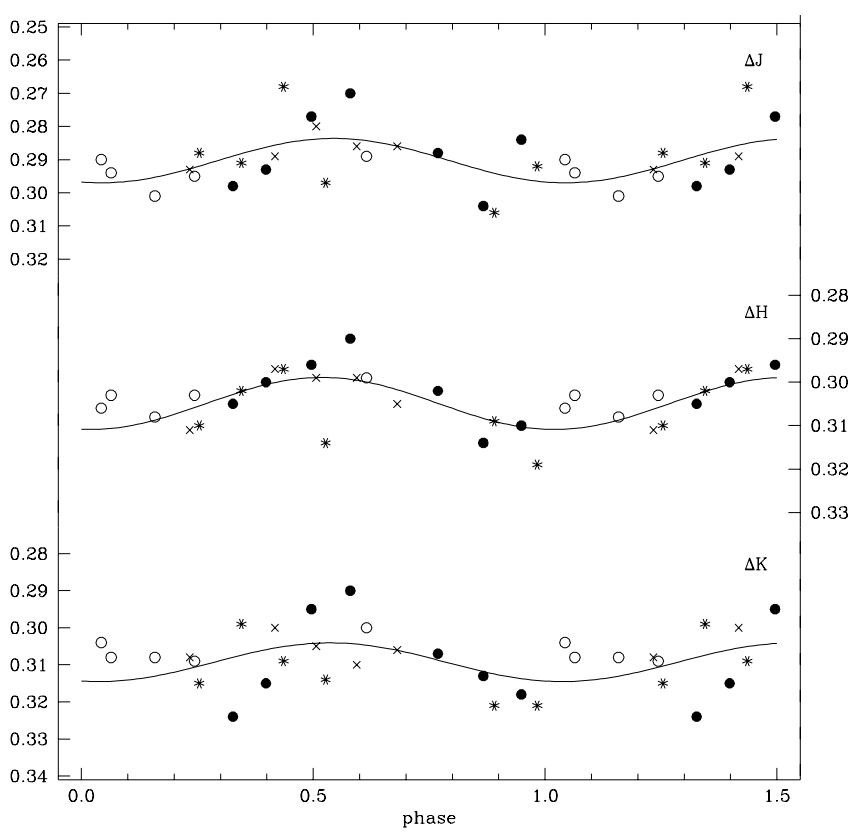

Fig. 10. Infrared light curves of HD 170397. The phases are computed according to the ephemeris elements given in Table 3. The solid line is a least-square fit of the observations by Eq. (1) as described in the text

$$
H D 223640=H R 9031=108 \text { Aqr }=E T A q r
$$

The photometric variability of HD 223640 has been studied in the Strömgren system by Morrison \& Wolff (1971) who found a period of $3.73 \mathrm{~d}$. The light curves show quite the same behaviour in all filters with a fairly constant amplitude of about 0.06 mag peak to peak.

Megessier \& Garnier (1972) contributed a few photometric data again in the Strömgren system and checked the spectroscopic variability; these authors found the $\mathrm{Ti}$ and Sr lines strongly variable and the lines of Fe constant. The variation of $\mathrm{Ti}$ correlates with the photometric variation, Ti being strongest when the star is brighter. Later Megessier (1974, 1975) interpreted all the variations in terms of the oblique rotator taking also into account the sign changes of the longitudinal magnetic field as determined by Babcock (1958).

Recent photometric observations have been carried out by North \& Burnet (1991), which have been supplemented with new magnetic field observations (North et al. 1992), and have led to an unambiguous and more precise value of the rotational period. In their detailed study of HD 223640 North et al. (1992) have confirmed the reversal of the effective magnetic field mentioned by Babcock (1958) and the nearly equatorial surface distribution of CrII and FeII, while TiI is mainly concentrated in patches at intermediate latitudes.

The infrared light curves of HD 223640 are plotted in Fig. 12 versus the phase computed by means of North et al. (1992) ephemeris elements listed in Table 3. From Fig. 12 we see that the light curves in all filters are in phase with each other and show the same simple-wave behaviour with 


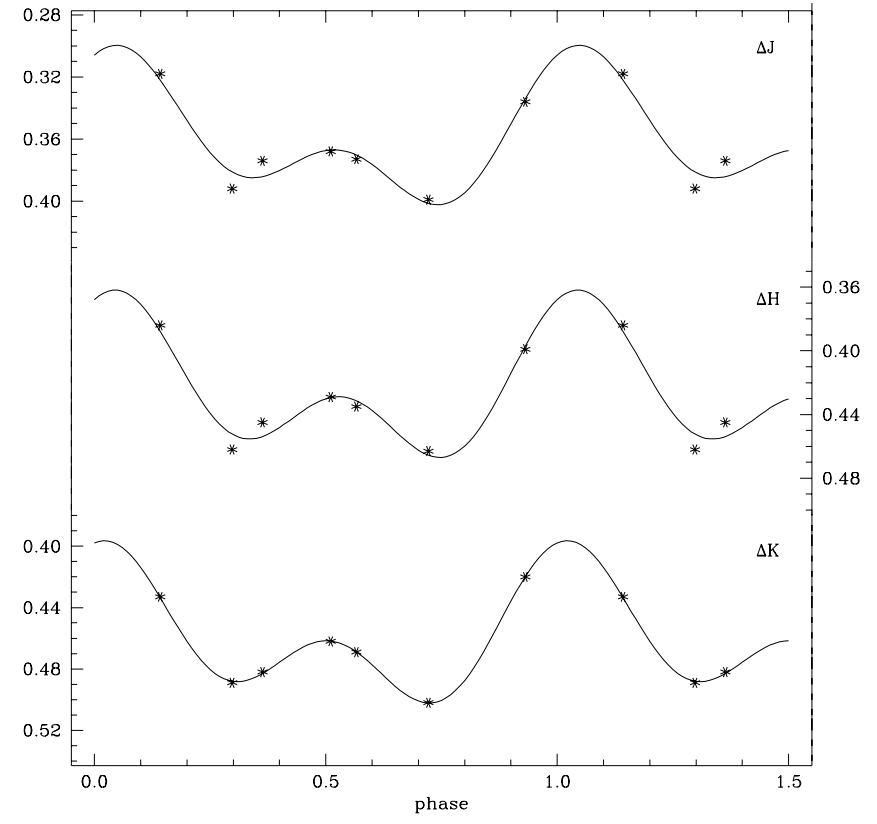

Fig. 11. Infrared light curves of HD 187473. The phases are computed according to the ephemeris elements given in Table 3. The solid line is a least-square fit of the observations by Eq. (1) as described in the text

amplitudes of the order of $0.02 \mathrm{mag}$ but with a quite large dispersion.

\section{Discussion and conclusions}

We have presented near infrared photometric observations of the SiSrCrEu subgroup CP2 stars HD 10783, HD 12447, HD 74521, HD 90044, HD 119419, HD 125630, HD 147010, HD 166469, HD 170397, HD 187473, and HD 223640.

Near infrared variability has been shown to be present in nearly all the twelve stars studied: however, in the case of HD 10783, HD 12447, HD 116458, HD 147010, HD 166469, HD 170397, and HD 223640 the variations do show a small amplitude and a large dispersion. The star HD 187473, which is one of the largest amplitude variables among CP2 stars, shows in the infrared the largest observed amplitude, although smaller than in the visible.

The typical trend of CP2 stars to present smaller amplitude light variations at increasing wavelength is confirmed: the amplitudes in the near infrared are smaller than in the visible and the variations tend to be in phase with each other in all filters and, at least for the few stars for which we could do contemporaneous uvby photometry, the variations are also in phase at least with the $y$ variation.

The origin of light variations in the ultraviolet and visible part of the spectrum is still unclear, only qualitative considerations have been made based on the assumption that elements are not homogeneously distributed over the surface. Leckrone et al. (1974) and Leckrone (1976), pointing out that $\mathrm{CP}$ stars are flux deficient in the ultraviolet if

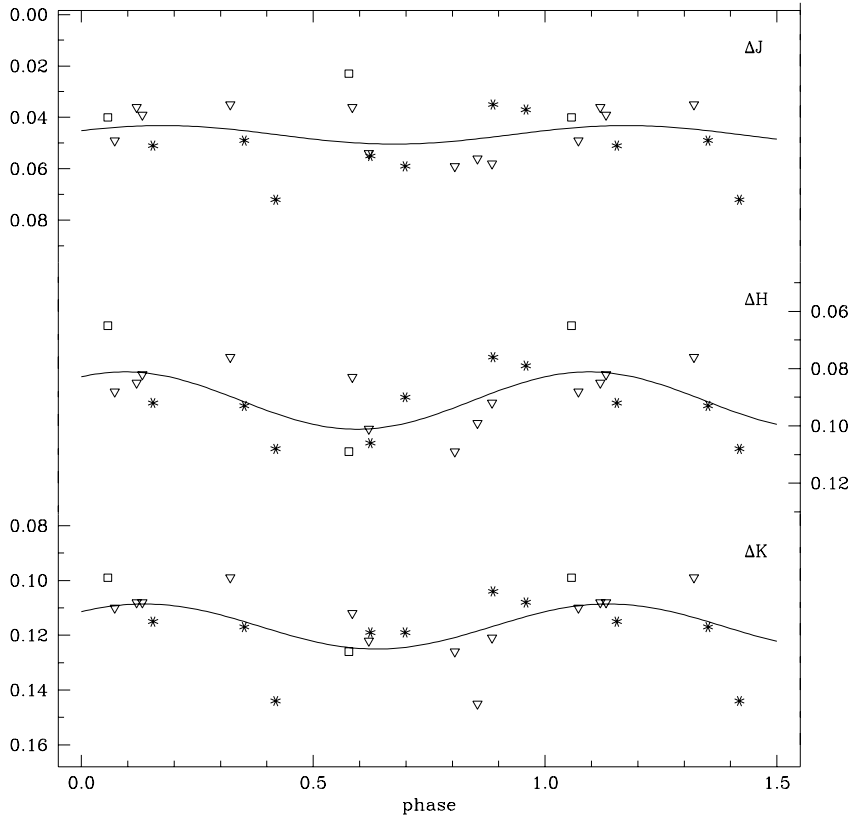

Fig. 12. Infrared light curves of HD 223640. The phases are computed according to the ephemeris elements given in Table 3. The solid line is a least-square fit of the observations by Eq. (1) as described in the text

compared to normal stars having the same Balmer jump, have suggested the presence of a greatly enhanced ultraviolet line opacity source, distributed more or less uniformly over the entire Balmer continuum region, and the redistribution of the absorbed UV flux longward of the nullwavelength region, that is the wavelength region with no observed variation.

According to Babcock (1958) and Deutsch (1958), Rare Earths and Fe are mainly concentrated in the positive magnetic pole region, while $\mathrm{Cr}$ and $\mathrm{Sr}$ are concentrated at the negative one. In a previous paper (CKL) we investigated the effects of high metallicity at the near infrared wavelengths. Because of the numerous metallic absorption lines, the blanketing mechanism steepens the temperature gradient and redistributes the flux from the ultraviolet, where the metallic absorption is strongest, to longer wavelengths, yet conserving the effective temperature. Since the atmospheres of CP stars show locally inhomogeneous metal distributions, the stellar rotation should communicate these optical depth variations as infrared variability. CKL have shown that a Kurucz model atmosphere with a metal content ten times the solar one could explain a three percent variation in the near infrared brightness, which is the typically observed value. The fact that the near infrared variations are in phase with those in the visible, or at least with those in $y$, may be an indication that the same mechanism is responsible for both.

Acknowledgements. We would like to thank Dr. P. Bouchet and R. Vega Tello for their help and advice during the observations and reductions. We are also grateful to the referee 
whose comments have contributed to a better presentation of the paper.

\section{References}

Adelman S.J., Pyper D.M., 1979, AJ 84, 1726

Albrecht R., Jenkner H., Weiss W.W., Wood H.J., 1977, A\&A 58,93

Babcock H.W., 1958, ApJS 3, 141

Bohlender D.A., Landstreet J.D., 1991 (priv. comm. to Mathys 1991)

Bohlender D.A., Landstreet J.D., Thompson I.B., 1993, A\&A 269, 355

Bonsack W.K., 1974, PASP 86, 408

Borra E.F., Beaulieu A., Brousseau D., Shelton I., 1985, A\&A 149,266

Borra E.F., Landstreet J.D., 1980, ApJS 42, 421

Bouchet P., 1989, Infrared Photometry, E.S.O. Operating Manual No. 11

Bouchet P., Manfroid J., Schmider F.-X., 1991, A\&AS 91, 424

Brown D.N., Landstreet J.D., Thompson I.B., 1981, A magnetic survey of Ap stars in young clusters - Preliminary results. In: $23^{\text {rd }}$ Liège Astrophys. Coll.. Upper Main Sequence Chemically Peculiar Stars. Universitè de Liège, p. 195

Catalano F.A., Leone F., 1993, A\&AS 100, 319

Catalano F.A., Renson P., 1984, A\&AS 55, 371

Catalano F.A., Renson P., 1988, A\&AS 72, 1

Catalano F.A., Renson P., 1997, A\&AS 121, 57

Catalano F.A., Kroll R., Leone F., 1991, A\&A 248, 179 (CKL)

Catalano F.A., Leone F., Kroll R., 1997, A\&AS (in press)

Catalano F.A., Renson P., Leone F., 1991, A\&AS 87, 59

Catalano F.A., Renson P., Leone F., 1993, A\&AS 98, 269

Deutsch A.J., 1958, Harmonic Analysis of the Periodic Spectrum Variables. In: Lehnert B. (ed.), Proc. I.A.U. Symp. 6, Electromagnetic Phenomena in Cosmical Physics. Cambridge University Press, Cambridge, p. 209

Deutsch A.J., 1947, ApJ 105, 283

Dworetsky M.M., Trueman M.R.G., Stickland D.J., 1980, A\&A 85,138

Glagolevskii Yu.V., Bychkov V.D., Iliev I.K., Romanyuk I.I., Chunakova N.M., 1982, Pis'ma AZh 8, 26

Hardie R.H., Reichmann E.J., Burke E.W.Jr., Hall D.S., 1990, J. Astrophys. Astron. 11, 277

Hensberge H., 1993, Long Term Variability in CP stars. In: Dworetsky M.M., Castelli F., Faraggiana R. (eds.), Proc. I.A.U. Coll. 138, Peculiar versus Normal Phenomena in AType and Related Stars, ASP Conf. Ser. 44, 547
Hensberge H., De Loore C., Zuiderwijk E.J., HammerschlagHensberge G., 1977, A\&A 54, 433

Hensberge H., Sterken C., Van Der Linden Th., HammerschlagHensberge G., 1978, A\&AS 34, 67

Hoffleit D., Jaschek C., 1982, The Bright Star Catalogue, $4^{\text {th }}$ revised edition, Yale University Observatory

Kameswara Rao N., Rajamohan R., 1982, Inf. Bull. Var. Stars 2121

Lanz T., Mathys G., 1991, A\&AS 90, 365

Leckrone D.S., 1976, Properties of Ap stars in the Ultraviolet. In: Weiss W.W., Jenkner H., Wood H.J. (eds.), Proc. I.A.U. Coll. 32, Physics of Ap Stars, Universitätssternwarte, Wien, p. 465

Leckrone D.S., Fowler J.W., Adelman S.J., 1974, A\&A 32, 237

Maitzen H.M., Wood H.J., 1977, A\&A 58, 389

Manfroid J., Mathys G., 1985, A\&AS 59, 429

Manfroid J., Renson P., 1980, Inf. Bull. Var. Stars 1824

Manfroid J., Renson P., 1983, A\&AS 51, 267

Manfroid J., Renson P., 1994, A\&A 281, 73

Manfroid J., Sterken C., Bruch A., et al., 1991, First Catalogue of stars measured in the Long-Term Photometry of Variables Project 1982-1986, E.S.O. Scientific Report No. 8 Mathys G., 1991, A\&AS 89, 121

Mathys G., Manfroid, J., 1985, A\&AS 60, 17

Megessier C., 1974, A\&A 34, 53

Megessier C., 1975, A\&A 39, 263

Megessier C., Garnier R., 1972, ApL 11, 113

Morrison N.D., Wolff S.C., 1971, PASP 83, 474

North P., 1982, Inf. Bull. Var. Stars 2208

North P., 1984, A\&AS 55, 259

North P., Burnet M., 1991, Inf. Bull. Var. Stars 3635

North P., Brown D.N., Landstreet J.D., 1992, A\&A 258, 389

Preston G.W., Stepien K., 1968, ApJ 154, 971

Rakos K.D., Fiedler W., 1978, A\&AS 31, 83

Renson P., 1978, Inf. Bull. Var. Stars 1391

Renson P., Gerbaldi M., Catalano F.A., 1991, A\&AS 89, 429

Steinitz R.W., 1964, Bull. Astron. Inst. Netherlands 17, 504

Stepien K., 1968, ApJ 154, 945

Thompson I.B., Brown D.N., Landstreet J.D., 1987, ApJS 64, 219

Van Genderen A.M., 1964, Inf. Bull. Var. Stars 76

Van Genderen A.M., 1965, Bull. Astron. Inst. Netherlands 18, 67

Van Genderen A.M., 1967, Bull. Astron. Inst. Netherlands 19, 80

Van Genderen A.M., 1971, A\&A 14, 48

Winzer J.E., 1974, Thesis, University of Toronto

Wood H.J., Campusano L.B., 1975, A\&A 45, 303 